\title{
The coronary sinus and adjacent structures in human adult hearts: morphometrical cadaveric anatomy
}

\author{
Eugeniya G. Dmitrieva ${ }^{1 \mathrm{~A}, 2 \mathrm{~B}}$, Anton A. Gaponov ${ }^{1 \mathrm{~A}}$, Sergey L. Khatsko ${ }^{3 \mathrm{~B}}$, Andrei A. Iakimov ${ }^{1 \mathrm{~A}, 2 \mathrm{~B}}$ \\ ${ }^{1}$ Department of Human Anatomy \\ ${ }^{2}$ Department of Medical Biochemistry and Biophysics \\ ${ }^{3}$ Anatomical and Physiological Experimental Laboratory \\ ${ }^{A}$ Ural State Medical University, Ekaterinburg, Russian Federation \\ ${ }^{B}$ Ural Federal University, Ekaterinburg, Russian Federation
}

\section{SUMMARY}

The aim of this study was to explore the anatomy and morphometry of the coronary sinus in hearts of adult humans, clarify position of the sinus to left atrium, left fibrous ring and adjacent arteries and describe topographic variants for adjacent anatomy of the coronary sinus in hearts of various types of coronary dominance. We studied 40 hearts weighing 200-420 g, obtained from patients who died from non-cardiac causes. We dissected 30 non-injected macroscopic specimens preserved into 4\% PBS formalin to reveal and measure coronary sinus and neighboring coronary arteries. The rest 10 hearts we used for preparing histological slices from the points of the beginning of the sinus, in the middle, and near the "crux cordis". We measured large and small diameters, circumference, wall thickness of the coronary sinus, as well as explored relative position of the coronary sinus, adjacent arteries, left atrium and left fibrous ring.

Corresponding author:

Andrei A. Iakimov, MD, PhD, Associate Professor. Department of Human Anatomy, Ural State Medical University, 20A Onufrieva street, Ekaterinburg, Russian Federation. Phone: +7 (904) 54492 82. E-mail: andrei071997@gmail.com
The reference range of coronary sinus length was $33.76-48.4 \mathrm{~mm}$. The median $(\mathrm{Me})$ of the circumference of the coronary sinus increased from $13.87 \mathrm{~mm}$ at the beginning of the sinus to $23.99 \mathrm{~mm}$ at the "crux cordis". In some specimens, the cross section of the sinus looked flattened in the anteroposterior direction; in others, it appeared to be flattened basoapically. We distinguished two anatomical patterns of the coronary sinus: subepicardial and intramyocardial. The first we proposed to term myocardialless or, simply, "naked", and the second type we named muscularized. We proposed to divide the latter into partially and completely muscularized. Lack of adventitia between the myocardial sheet and tunica media of the coronary sinus allowed us to consider myocardial sleeves as an integral structure of the intramyocardial sinus wall. The wall thickness value of the coronary sinus at the point of origin, in the middle, and at the point of its turning anterosuperiorly toward the right atrium had no differences, but significantly depended on the myocardial cover and on the position of the

Submitted: July 19, 2020. Accepted: July 9, 2021

https://doi.org/10.52083/BNUM8219 
coronary sinus in the left atrioventricular sulcus. The distance from the sinus to the posterior wall of the left atrium was 0.15-7.66 mm (Me 0.91 mm); the distance to the left fibrous ring was 2.9-13.7 mm (Me $7.9 \mathrm{~mm}$ ). We found four variants of topographic and anatomical relations of the sinus and its adjacent arteries. Commonly, arteries were located below the coronary sinus. The ranges of variability of morphometric values of the coronary sinus and neighboring structures may be considered as reference values of the anatomy of the coronary sinus in the normal human adult heart.

Key words: Gross anatomy - Heart - Cardiac veins - Coronary sinus - Coronary arteries - Left atrium

\section{INTRODUCTION}

Normal anatomy abounds of studies regarding cardiac morphology, but some structures of the heart still remain incompletely described. One structure that lacks detailed description is the coronary sinus (CS). CS is the greatest vein that drains venous blood from the most part of the cardiac walls. CS starts at the confluence point of the great cardiac vein and the oblique vein of the left atrium (LA), runs in the left posterior part of the atrioventricular groove, and opens into the right atrium. Clinically, CS is a cardiac structure, which is mainly the focus of interventional electrophysiological approaches, such as pacemaker lead placement for cardiac resynchronization therapy or radiofrequency ablation (Plass et al., 2008). Myocardial continuity from the right atrium through CS toward the posterior wall of LA may influence interatrial pacing and provide atrial fibrillation (Kasai et al., 2001; Habib et al., 2009). The coronary artery in the left posterior atrioventricular groove may cross the CS, and such anatomical pattern could represent an increased risk for compression of the artery after device implantation (Plass et al., 2008). The anatomical variants of relations of the left circumflex artery and the CS determine the safety of mitral annuloplasty as an occlusion of the artery, and its consequences might occur in some cases (Młynarski et al., 2013). Arguably, the most important clinical application is in the risk of compression of the neighboring artery, with the subsequent development of local myocardial ischemia. Being inserted into CS, a catheter may press the artery to the upper edge of the inferior wall of the left ventricle. The greatest risk of artery compression relates to the location of artery below or in front of the CS, between the CS, mitral annulus and the posterior wall of the LA (Sorgente et al., 2008). Thus, knowledge of the anatomy of the cardiac vessels and any variations are important for surgeons (Młynarski et al., 2013).

A multitude of studies, investigating various aspects of the morphology of CS, both provided new data and generated a lot of contradictions in describing of the morphology, morphometry and location of CS. Myocardial loops and sleeves over and around the CS have been described (Chauvin et al., 2000; Barcelo et al., 2004), but there is no clarity as to whether the myocardium is a layer of the sinus wall or should be considered as an element external to the CS. Morphometrical data of CS walls, with and without myocardial sleeves, are particularly lacking in literature. To our knowledge, there have been no detailed morphometric studies describing location of the CS of various patterns in relation to adjacent structures such as LA, left fibrous ring (LFR) and coronary arteries. The relationship of coronary dominance to various topographical forms of CS has not yet been evaluated. The aim of this study was to explore the anatomy and morphometry of the CS in hearts of adult humans, clarify the position of CS in relation to the left atrium, left fibrous ring and adjacent arteries, and describe topographic variants for adjacent anatomy of CS in hearts of various types of coronary dominance.

\section{MATERIALS AND METHODS}

\section{Design of the study}

The design of the study is a cross-sectional (transverse) observational, single blind (masked) study. Researchers who directly worked with the anatomical and histological material (E.G.D., A.A.G., S.L.K.) had no information about the anamnesis and personal data of the deceased persons. 


\section{Ethical Compliance}

Anatomical material was obtained during the period 2017-2019 from the pathomorphological departments of the University clinics. Hearts belonged to the deceased whose bodies nobody claimed for burial, so we had no possibility for getting informed consent. The study project has been approved by the local ethics committee of the Ural State Medical University (Yekaterinburg, Russia).

We screened a total of 73 adult human hearts which have been obtained for criteria as follows. Inclusion criteria: 1) hearts obtained from cadavers that were not claimed for burial, 2) death from non-cardiac diseases, 3) normal left-sided position of the heart in the thorax, 4) heart weight 200-420 g. 17 hearts were excluded for the follow criteria: 1) abnormalities of cardiac chambers or septa (1 heart), 2) any evidence of previous cardiac surgery (2 hearts), 3) gross pathological changes of the heart or cardiac blood vessels (14 hearts). We randomly divided 56 selected hearts into two groups. The first group $\left(\mathrm{n}_{1}=30\right)$ were used for gross anatomic investigation. The other 26 hearts were screened histologically for any pathological processes in the heart wall. 16 hearts appeared to be unsuitable for normal anatomical study, mainly due to myocardial and perivascular fibrosis, or inflammatory. Thus, the group for microscopic investigation ( $\mathrm{n}_{2}$ ) included 10 hearts. Eventually, we studied a total of 40 hearts with the aim of this research. The specimens of both groups were immersed and preserved into 4\% PBS formalin. The specimens for gross anatomy investigation were preliminary washed from blood clots. Before studying, the specimens of the first group were water-soaked for several days and dried at the air. Then we weighed the hearts used EJ-610 scales (A\&D Co LTD, Japan). Following preliminary examination, epicardium and subepicardial fatty tissue were carefully removed to visualize the course of the CS, its tributaries, coronary arteries and their branches. With the caliper (precision $0.1 \mathrm{~mm}$ ), we measured the length of the ventricular heart complex (VHC) from the apex of the heart to the origin of the aorta at the atrioventricular sulcus. The width of the VHC was evaluated as a maximum distance between the left and right pulmonary surfaces of the heart along atrioventricular sulcus (Fig. 1a, b). The term "ventricular heart complex" meant the part of the heart that included both ventricles, their lateral walls, the ventricular septum, as well as aortic and pulmonary roots. We used the specimens of the first subgroup also for exploring the relative position of the CS, its tributaries, and adjacent arteries which were found to be in the left

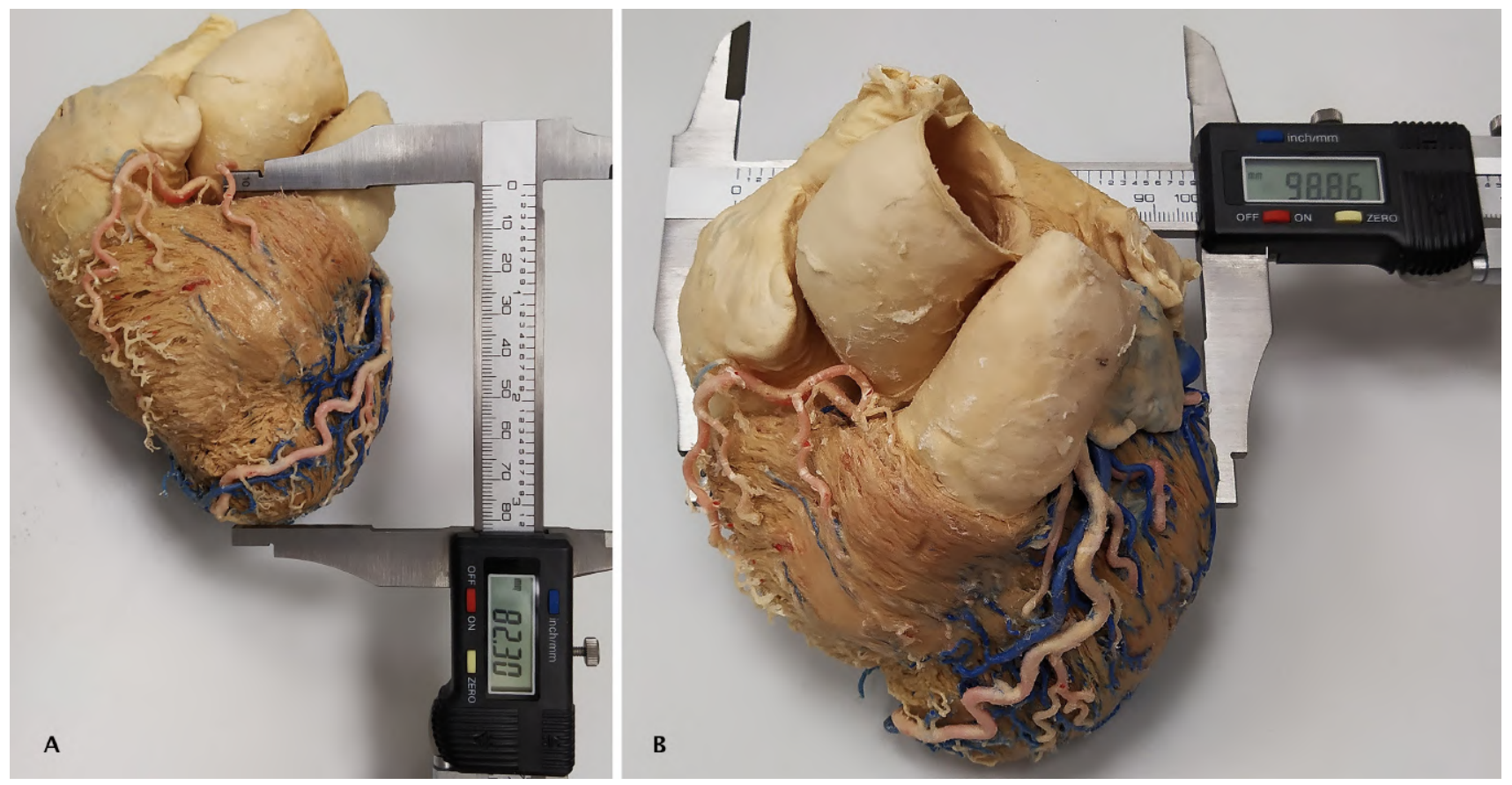

Fig. 1.- Morphometry of the length (a) and width (b) of the ventricular heart complex. Plastinated specimen used for demonstration of the method only. 
posterior atrioventricular groove. We determined the type of ventricular coronary dominance (dominance of the right coronary artery, balanced circulation and dominance of the left coronary artery) based on the posterior interventricular branch belonging to the system of the left or right coronary artery (LCA, RCA).

We cut pieces of the heart for histologic study a) from the confluence of the great cardiac vein with the oblique vein of the LA (beginning of the CS), b) from the middle point of the CS length and 3) from the medial part of the CS immediately before its turning antero-superiorly towards the right atrium (Fig. 2). The fixed tissue blocks were washed with tap water, dehydrated through an ethyl alcohol series, cleared in xylene and embedded in paraffin. Sections of 5-7 $\mu \mathrm{m}$ thickness were made transverse to the axis of CS and stained with Ehrlich hematoxylin and eosin, as well as Van Gieson technique with aqueous picric acid solution and aqueous acid fuchsin solution. We have made a total of 60 histological specimens (20 from each location). Olympus CX31RTSF trinocular histological microscope (Japan) with TOUPCAM U31SPM18000KPA digital camera, and ADF Image Capture 4.7 (2019) software were used. Full histotopographic views were composed with the software option "stitching".
The thickness of the anterior, posterior, superior and inferior wall of the CS was measured in all of three places where the tissue pieces for histologic slices were taken from (the beginning, middle and medial part of the CS). We measured each wall three times: in the thinnest place, thickest place and in a section of medium thickness, and the results presented as the arithmetic mean of three measurements. In the presence of myocardial sleeves of the CS, we measured wall thickness from the CS endothelium to the inner layer of the sleeve (without myocardium), and then measured from the CS endothelium to outside surface of the sleeve (including the myocardium). In histotopograms, we measured large (maximal) and small (minimal) diameters between the opposite points of the CS endothelium. By means of the option "curve line", we drew a line along the endothelium of the CS and the length of the line was considered as the circumference length. In order to compare the cross-sectional diameter of the CS with the data of other authors who considered the cross section of the CS to be round a priori, we applied the formula $L=2 \pi$. For studying adjacent anatomy of the CS, we measured distances from the most external adventitial fibers of CS to those of the neighboring coronary artery, to the most external dense collagen fibers of the LFR, and

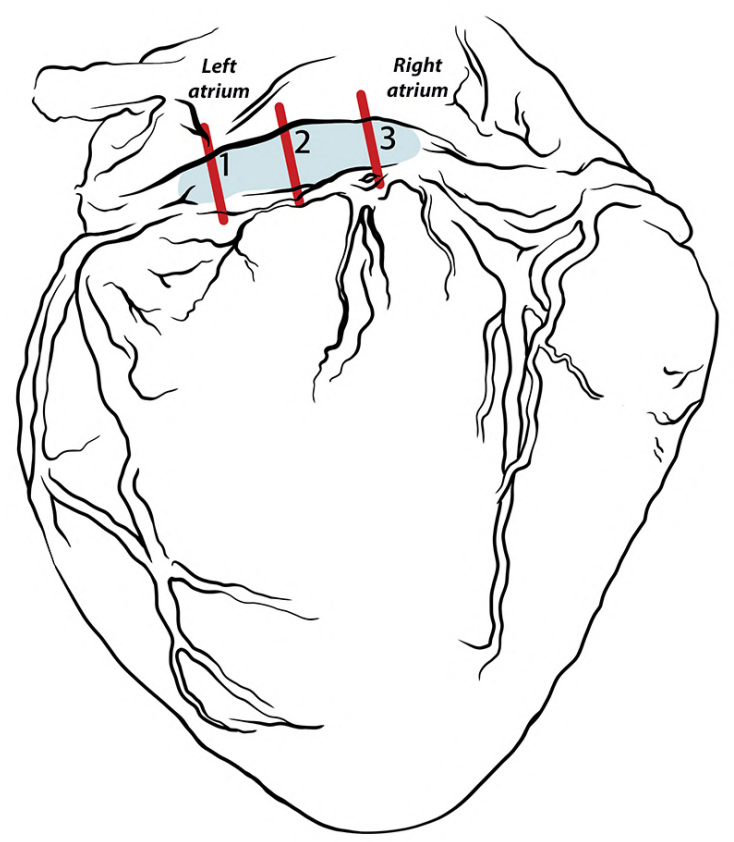

Fig. 2.- Sites for cutting heart tissue for histological sections. Red lines mark the incisions through the origin (1), middle (2) and pre-ending site (3) of the coronary sinus. Posterior view. 
to the nearest point of the external myocardial layer of the posterior wall of the LA. Besides, we assessed the presence and number of arteries in the left part of the posterior atrioventricular groove, as well as location of the arteries relative to the CS.

We used the Statistica 13.0 (StatSoft Inc, USA) software. The distribution of values was evaluated using the Shapiro-Wilk test. If the distribution appeared to be abnormal at least in one of the compared groups, the results were presented as extreme values (minimum, maximum), percentiles (p25, p75), and medians (Me). For multiple comparisons, we used the KruskalWallis $\mathrm{H}$ test. If the criterion revealed statistically significant differences, we proceeded to the pairwise comparison procedure with MannWhitney U-test. For the correlation analysis, the Spearman correlation coefficient (Rs) was used. The significance level $\alpha$ was $0.05(p<0.05)$.

\section{RESULTS}

\section{Anatomical parameters of the heart and coronary sinus}

The Table 1 shows the values of the length and width of the VHC as well as the value of the heart weight. The length of the CS was found to range from 13.7 to $50 \mathrm{~mm}$ (Me 34.25). The length of the CS correlated with the width (Rs 0.38) and the length of the VHC (Rs 0.30). No correlation was found to be between the length of the CS and the heart weight (Rs 0.19).

We have distinguished CS for two groups: short $(\mathrm{Me}=22.9 \mathrm{~mm})$ and long $(\mathrm{Me}=37 \mathrm{~mm})$ sinuses (Fig. 3). Medians of those groups showed significant differences $(p<0.001)$. Sinuses of various lengths differed in the number of tributaries: one posterior vein of the left ventricle flowed into short sinuses, and two or three of these veins opened into long ones. The middle cardiac vein was found to be the largest tributary of both short and long CS. This vein always opened into the CS shortly before its turning antero-superiorly toward the right atrium. Both diameters of CS as well as circumference of CS varied over a wide range (Table 2). The circumference of the CS was shorter $(U=23, p=0.045)$ at its origin than at the "crux cordis", where CS turned toward the right atrium (Table 2).

In some specimens, the CS appeared to be flattened both in macroscopic specimens and histotopograms. One group of specimens showed the CS flattened in the anteroposterior direction. The large diameter of the CS was parallel to the posterior wall of the LA, and the anterior and

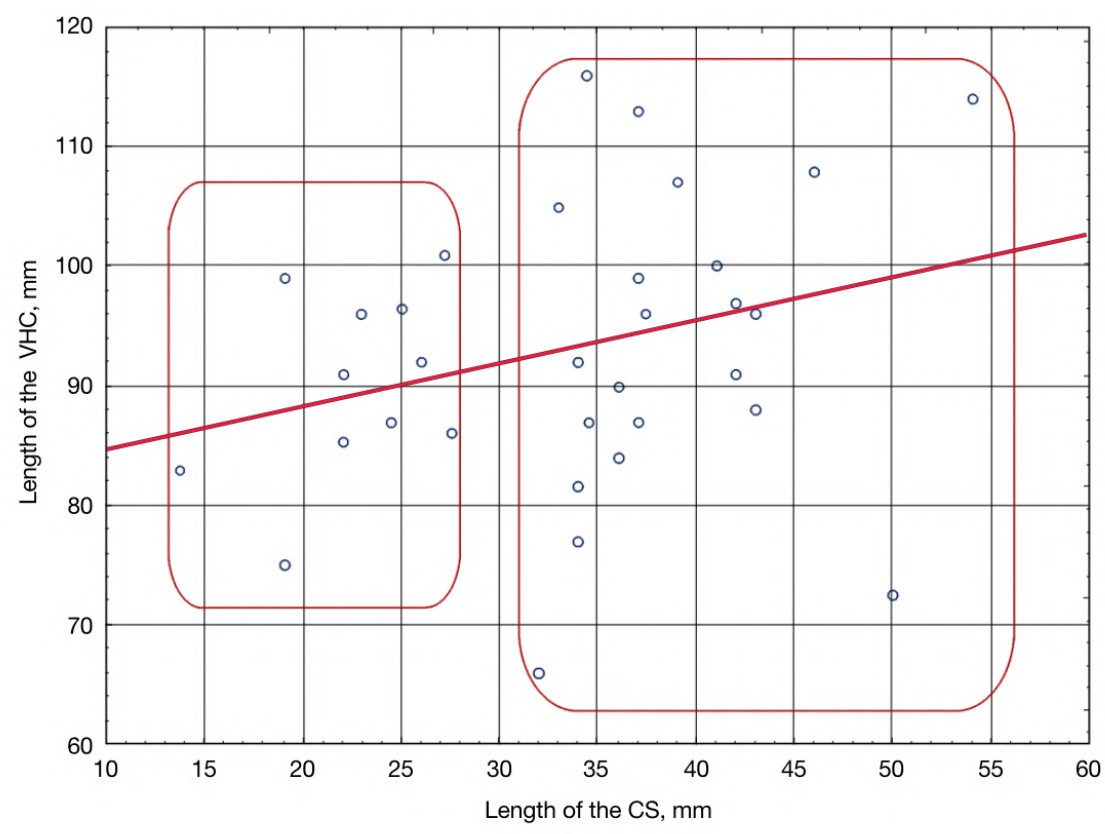

Fig. 3.- Length values of the short (left cluster) and long (right cluster) coronary sinuses (CS) and regression fit of the values on the length of the ventricular heart complex (VHC). 
posterior walls were longer than the superior and inferior ones. Contrariwise, other sinuses seemed to lie in the coronary groove having been flattened in the superoinferior direction. The superior and inferior walls of those CS were the longest. The large diameter of such sinuses was perpendicular to the posterior wall of the LA (Fig. 4a, b).

When studying histotopograms, it was found that in most cases the CS partially or completely passed through the myocardial sleeves, which were formed by the myocardium of the posterior wall of the LA (Figs. 5, 6a, b). The absence of adventitia, which would separate the sleeve from the media, allowed to consider this structure, if any, as an integral part of the wall of the CS. Myocardial sleeves significantly affected the variability of the CS wall thickness (compare Fig. 7a and 7b). When comparing the CS wall thickness values, measured with sleeves, in three locations, differences in the thickness values of the anterior $(\mathrm{H}=9.85 ; \mathrm{p}=0.007)$ and the posterior walls $(\mathrm{H}=11.33 ; \mathrm{p}=0.004)$ were revealed. One group of specimens showed the myocardial sleeve surrounded the entire lateral half of the CS while the others found myocardium to be at the turning of the CS anterosuperiorly. The sleeve thickened the anterior wall in the middle of CS, and the posterior wall at the turning of the CS toward the right atrium. The thickness of the anterior wall increased on average 2.5 times: from $346 \mu \mathrm{m}$ at the origin of the CS to $875 \mu \mathrm{m}$ at its end $(\mathrm{U}=6$; $\mathrm{p}=0.003)$. The thickness of the posterior wall throughout the CS increased 1.9 times: from 468 $\mu \mathrm{m}$ to $910 \mu \mathrm{m}(\mathrm{U}=6 ; \mathrm{p}=0.003)$. Unlike anterior and posterior walls, nor superior neither inferior wall thickness changed significantly along the CS in the three locations.

We had measured four walls of the CS without sleeves in three locations, and we did not find to be significant differences in the thickness of the anterior $(\mathrm{H}=0.44, \mathrm{p}=0.80)$, posterior $(\mathrm{H}=0.66, \mathrm{p}$ $=0.72)$, superior $(\mathrm{H}=2.0, \mathrm{p}=0.37)$ and the inferior $(\mathrm{H}=2.49, \mathrm{p}=0.29)$ walls. Also, no differences were found between the thicknesses of different walls of the CS within the same location (Fig. 7b). The absence of statistically significant differences allowed to combine these data to calculate medians and percentiles. The median wall thickness of the CS was $525 \mu \mathrm{m}$ (p25-p75 = 364-839 $\mu \mathrm{m})$.

\section{Adjacent anatomy of the coronary sinus, left atrium, and the left fibrous ring}

The distance from the CS to the LFR (Me $=7.9$ $\mathrm{mm}$ ) and to the myocardium of the LA posterior wall $(\mathrm{Me}=0.91 \mathrm{~mm})$ varied widely (Table 3 ). Heading down to the LFR, the posterior wall of the LA shifted forward moving more and more away from the CS. As a result, the distance from the CS to the LFR was greater than the distance from the CS to higher sections of the LA posterior wall. This

Table 1. Data of the heart measurements.

\begin{tabular}{|l|l|l|}
\hline Parameter & Median & Extreme values \\
\hline Weight of the heart, g & 301 & $197 ; 419$ \\
\hline Length of the VHC, mm & 92 & $72 ; 113$ \\
\hline Width of the VHC, mm & 97 & $81 ; 121$ \\
\hline
\end{tabular}

VHC - ventricular heart complex

Table 2. Microanatomic morphometric parameters of the coronary sinus (CS), $\mathrm{mm}$.

\begin{tabular}{|c|c|c|c|}
\hline Place of measurement & Minimal diameter & Maximal diameter & Circumferential length \\
\hline Origin of the CS & $\begin{array}{l}0.17 \\
(0.02: 3.80)\end{array}$ & $\begin{array}{l}5.94 \\
(3.16: 7.70)\end{array}$ & $\begin{array}{l}13.87^{*} \\
(7.46: 29.63)\end{array}$ \\
\hline Middle point of the CS length & $\begin{array}{l}0.18 \\
(0.02: 1.53)\end{array}$ & $\begin{array}{l}6.34 \\
(3.64: 15.48)\end{array}$ & $\begin{array}{l}16.40 \\
(7.63: 35.00)\end{array}$ \\
\hline $\begin{array}{l}\text { Turning of the CS from the av-groove anterosupe- } \\
\text { riorly toward the right atrium }\end{array}$ & $\begin{array}{l}0.76 \\
(0.05: 2.61)\end{array}$ & $\begin{array}{l}9.13 \\
(3.17: 16.34)\end{array}$ & $\begin{array}{l}\text { 23.99* } \\
(10.49: 40.16)\end{array}$ \\
\hline
\end{tabular}

$(*)-\mathrm{p}<.05$ 
peculiarity of the CS with relation to LA and LFR, first of all, depended on the shape of the CS cross section and its orientation in the coronary groove (compare Fig. 4a and 4b). When comparing the distances at the beginning and in the middle of the CS, no differences were found either between the values of the distances from the CS to the LA ( $\mathrm{U}=$ $36 ; \mathrm{p}=0.29$ ) or between the values of the distances from the CS to the LFR ( $U=42 ; p=0.57)$. Hence, it follows that the anterior wall of the lateral half of the CS was always parallel to the posterior wall of the LA. The adjacent anatomy of the medial half of the CS, the atrial complex and the fibrous skeleton of the heart were more complicated. On its way to the opening into the right atrium, the medial part of the CS lay behind the postero-inferior portion of the posterior atrial groove, at the "crux cordis", which is the base of the inferior pyramidal space. Here, the posterior wall of the LA turned forward and inward, where it formed the left wall of that space, traditionally mistakenly referred to as the interatrial septum (Anderson et al., 2013). The posterior fragment of LFR also, following the posterior wall of the LA, was directed forward. As a result, no structures that could be attributed to the fibrous skeleton of the heart were revealed in front of the end section of the CS either by gross dissection or by studying histological specimens.
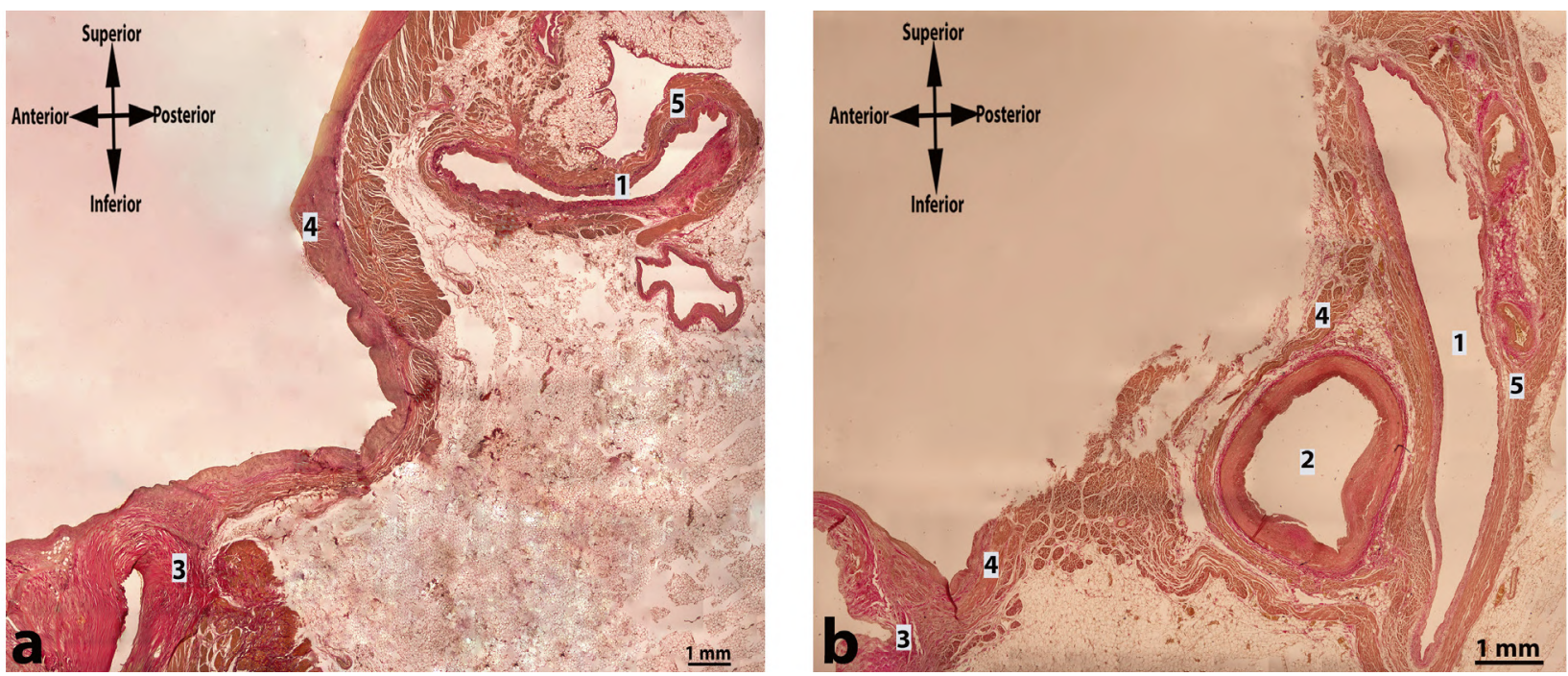

Fig. 4.- Anatomical patterns of the cross section of the coronary sinus (CS) in situ. a - the sinus is flattened in supero-inferior direction ("lying CS") $\mathbf{b}$ - the sinus is flattened in anteroposterior direction ("upright CS"). $\mathbf{1}$ - coronary sinus, $\mathbf{2}$ - coronary artery, $\mathbf{3}$ - left fibrous ring, $\mathbf{4}$ - posterior wall of the left atrium, $\mathbf{5}$ - myocardial sleeve of the coronary sinus. Van Gieson, x 40.

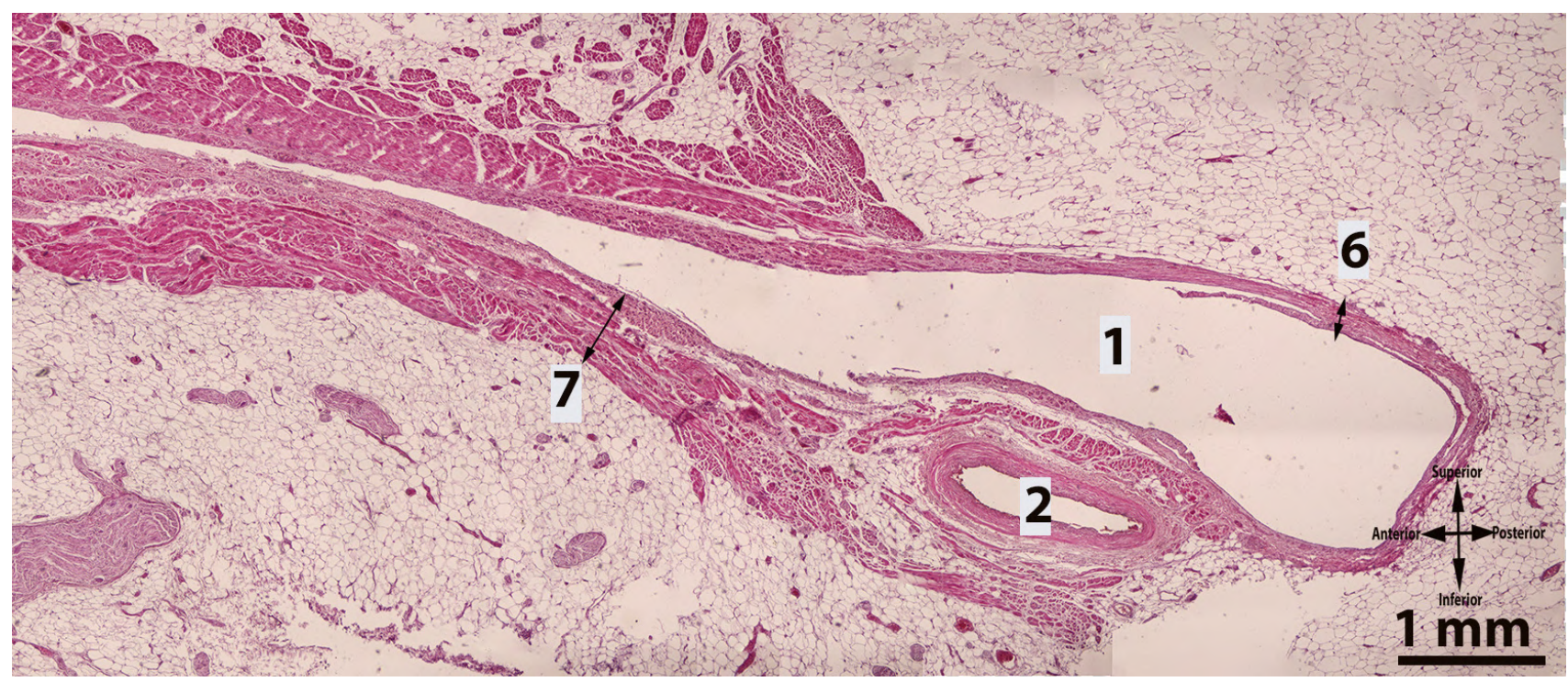

Fig. 5.- Partially muscularized coronary sinus. 1 - coronary sinus, 2 - coronary artery, 6 - coronary sinus wall without myocardial sleeve, 7 - coronary sinus wall with myocardial sleeve. Cross section. Hematoxylin \& eosin staining, $\mathrm{x} 40$. Scale bar $=1 \mathrm{~mm}$. 
Having passed behind the "crux", the CS opened in the right atrium through its inferoposterior wall. It was noteworthy that in all cases, passing in the region of the "crux", the CS bent forward, deviating from the atrioventricular groove by about $30-60^{\circ}$. As a rule, the place of bending coincided with the place where the middle cardiac vein opens into the CS.

\section{Coronary sinus and adjacent arteries}

The study of histological specimens showed that at the origin of the CS the arterial vessels closely adjoined to the CS in seven out of ten cases. In six specimens, the artery was located below (see Figs. $5,6 \mathrm{~b})$, in one case the artery ran in front and was separated from the CS by $0.8 \mathrm{~mm}$ thick myocardial loop from the layer of the atrial myocardium (see Fig. 6a). Myocardial loops around the arteries near the CS were discovered in three hearts studied with histologic technique and in two hearts dissected and explored macroscopically. At the middle of the CS, the artery was located at the bottom of the CS in five specimens, posteriorly to the CS in three specimens, and ran in front of the CS in one heart. At the basis of the inferior pyramidal space ("crux"), arteries contiguous with the CS were found in seven specimens. In three hearts from those seven, arteries closely adjoined to the medial wall of the CS. When comparing the distances from the artery to the CS in its beginning, in the middle and near the "crux", we have found no differences $(\mathrm{H}=1.18$; $\mathrm{p}$ $=0.55)$. The median of this distance was $0.73 \mathrm{~mm}$ (p25-p75 = 0.25-2,63 mm).

When studying CS and arteries on macroscopic specimens, we delineated four topographic variants for adjacent anatomy of these vessels (Fig. 8 a-d). In the first group of samples (10 of $30,33.3 \%)$, the circumflex branch of the LCA continued into the left marginal artery and did not reach the $\mathrm{CS}$, while the RCA or its circumflex branch came into contact with its anterior or inferior wall near the "crux" (Fig. 8a). In the second group (9 of $30,30 \%$ ), the left circumflex branch had the topography similar to the first group, while the terminal part of the RCA was adjacent to the CS throughout its entire length (Fig. 8b). In the third group, (4 of 30,13.3\%), the left circumflex artery was adjacent throughout the anterior or inferior wall of the CS (Fig. 8c). In the fourth group (7 of 30, 23.3\%), both the left circumflex branch and the terminal part of the RCA ran closely to the anterior or inferior wall of the CS (Fig. 8d).

26 hearts showed right coronary dominance, two hearts showed left dominance, and the
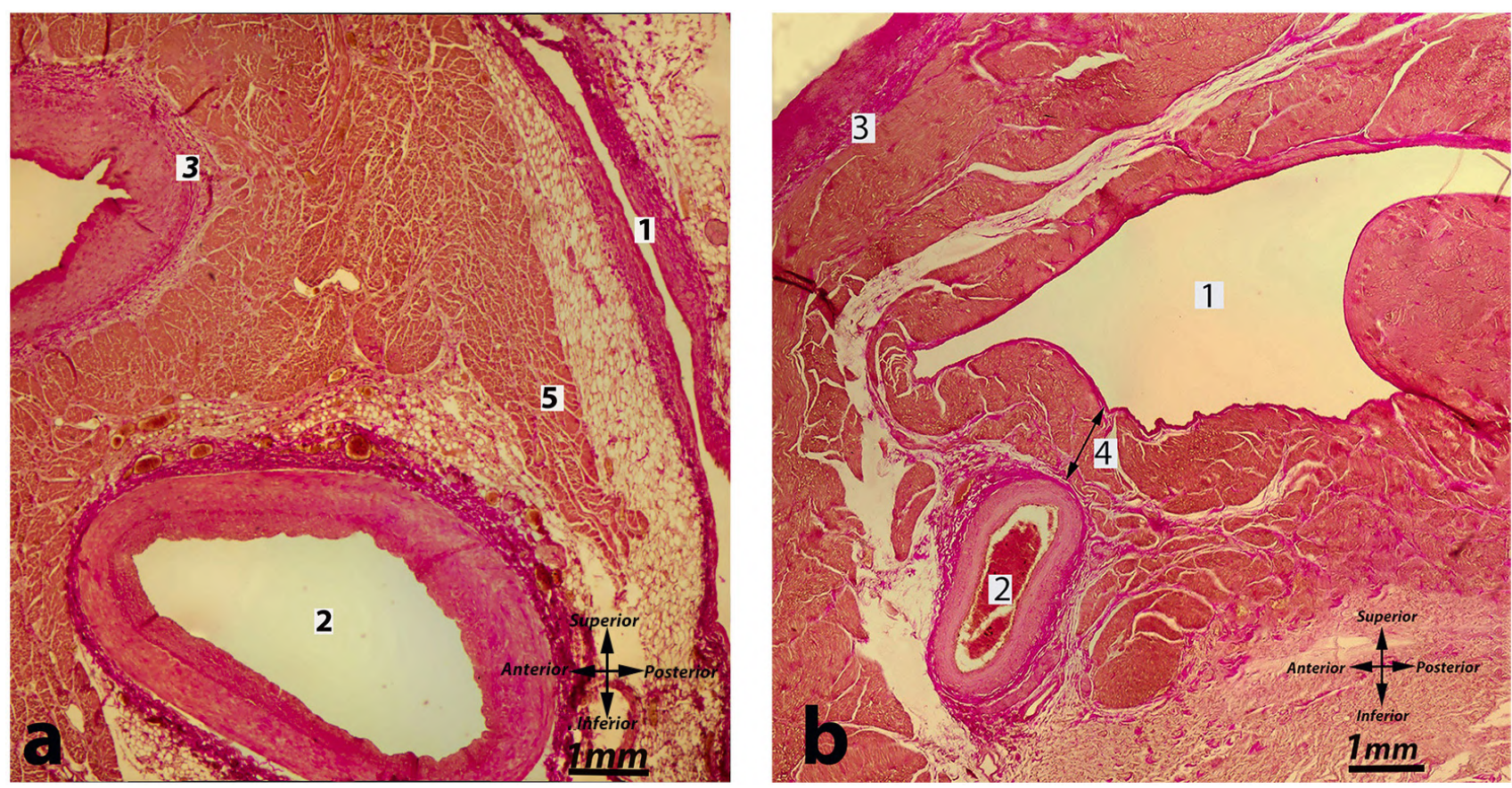

Fig. 6.- Anatomical variants of the coronary sinus (CS) wall. a: CS lies subepicardially and is flattened anteroposteriorly. b: CS lies intramyocardially 1 - coronary sinus, 2 - coronary artery, 3 - posterior wall of the left atrium, 4 - myocardial sleeve of the CS, 5 - periarterial myocardial loop. Van Gieson staining, $x 40$. Scale bars $=1 \mathrm{~mm}$. 
remaining two hearts had balanced circulation. In the hearts with left coronary dominance and balanced circulation, the CS followed the circumflex branch of the LCA. In those with right coronary dominance, RCA was adjacent to the CS in 19 specimens (63.4\%), which corresponded to the first and second variants of the adjacent anatomy of the blood vessel. In the remaining seven cases, the circumflex branch of the LCA and the RCA were adjacent to the initial and terminal parts of the CS. Thus, three variants of the vascular relationships of the CS were noted in right coronary dominance.

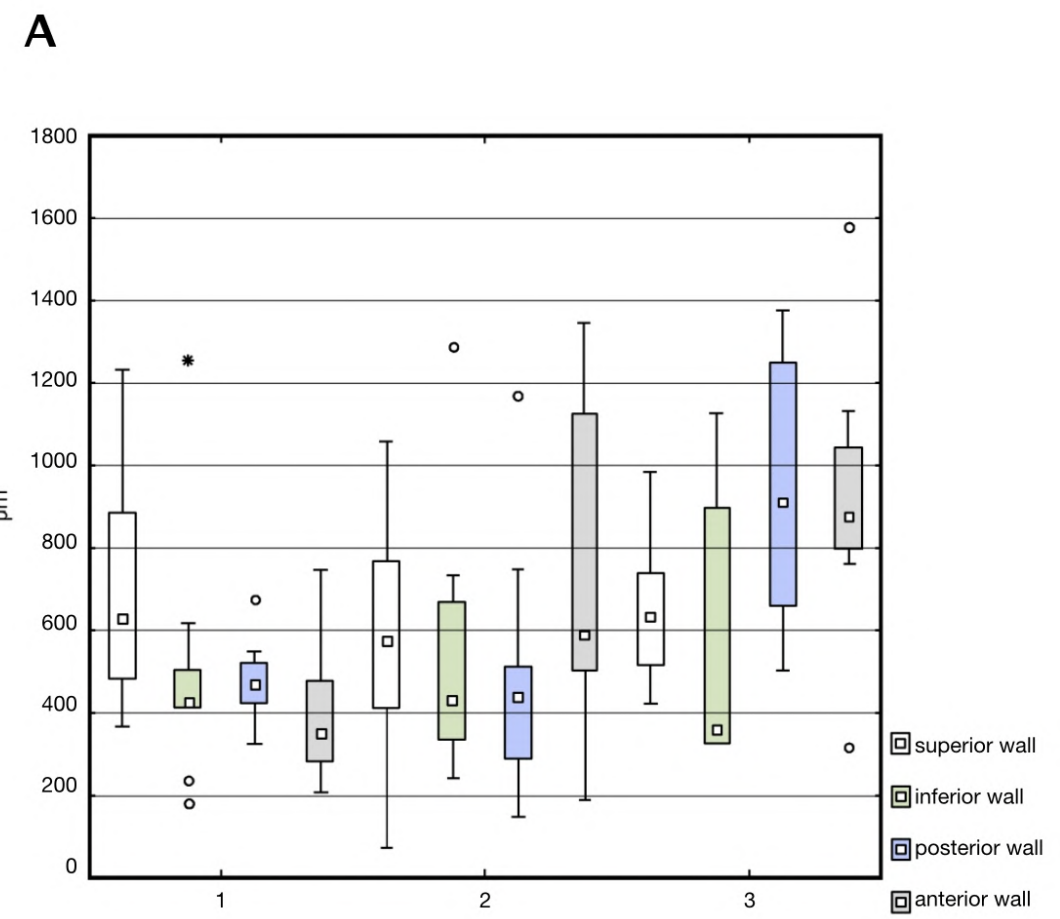

B

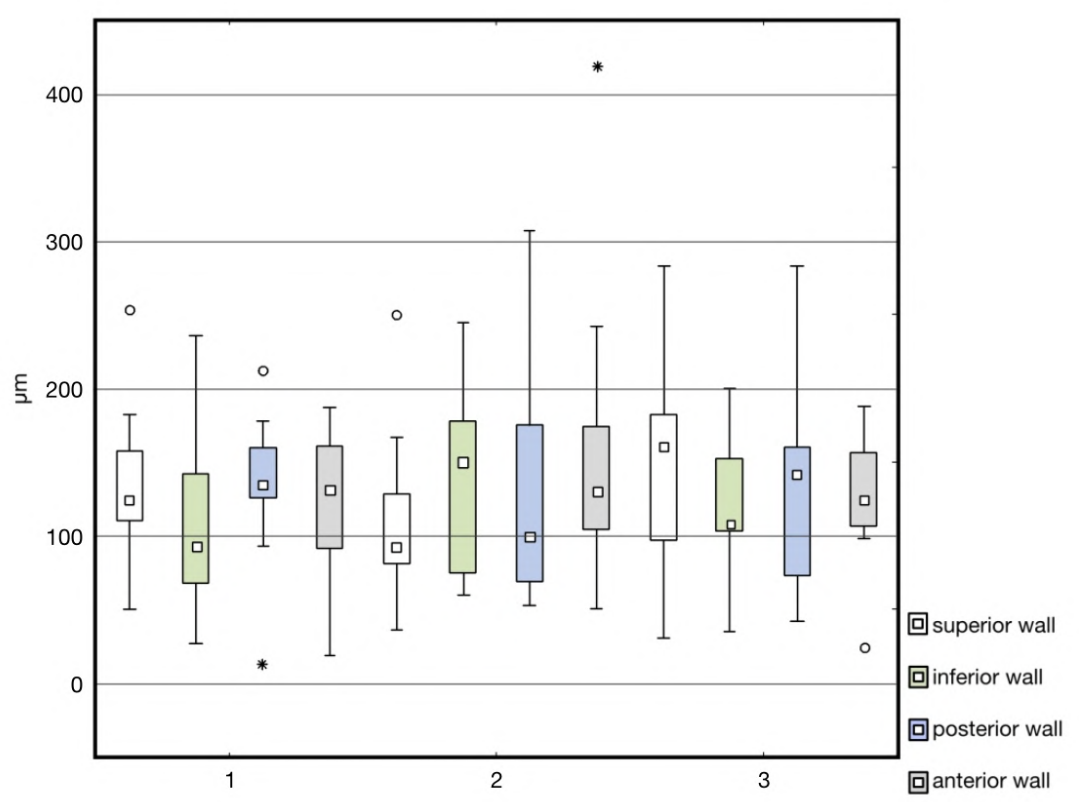

Fig. 7.- Thickness of the walls of the coronary sinus, measured with (A) and without (B) myocardial sleeve. Median. Box - p25-p75. Whiskers extreme values. 
Table 3. Distance from the coronary sinus (CS) to the left fibrous ring, and to the posterior wall of the left atrium, $\mathrm{mm}$.

\begin{tabular}{|l|l|l|}
\hline Place of measurement & Posterior wall of left atrium & Left fibrous ring \\
\hline Beginning of the CS & $1.57(0.17: 6.33)$ & $18.21(9.69: 32.45)$ \\
\hline Middle of the CS length & $2.04(0.10: 7.31)$ & $14.65(3.51: 25.37)$ \\
\hline
\end{tabular}
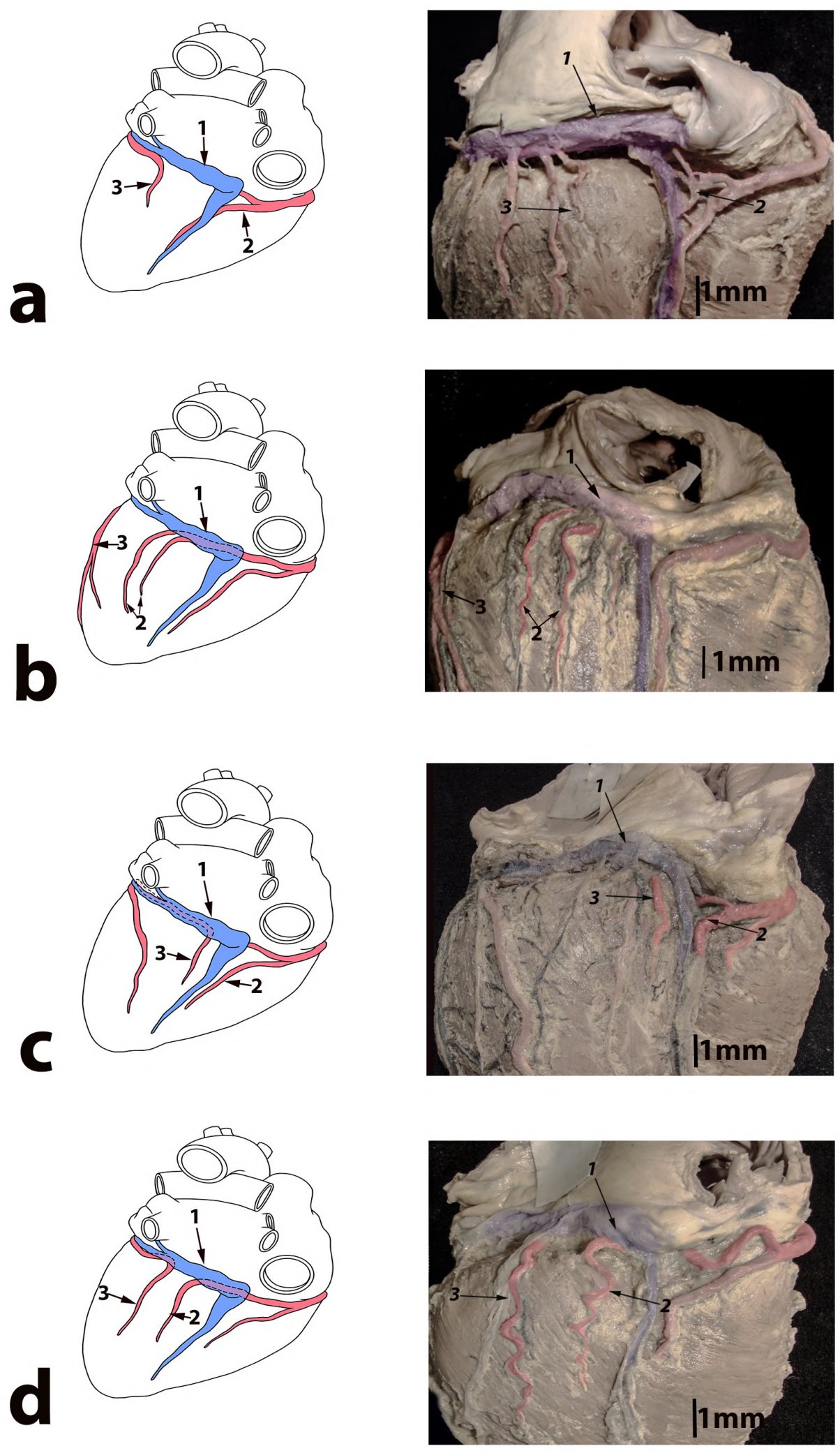

Fig. 8.- Topographical variants of the coronary sinus and coronary arteries. $\mathbf{a}$ - the $1^{\text {st }}, \mathbf{b}$ - the $2^{\text {nd }}, \mathbf{c}-$ the $3^{\text {rd }}, \mathbf{d}-4^{\text {th }}$ variant. Gross anatomical dissection of the non-injected vessels. We corrected colors of vascular images in the Photoshop photo editor. Blue showed the coronary sinus, red colored arteries from the systems of the right (2) and left (3) coronary arteries. 


\section{DISCUSSION}

\section{Morphometry of the coronary sinus}

In this study, we have explored anatomy and morphometry of the CS in normal human hearts both gross- and micro-anatomically. We identified the relationship between the length of the CS and the length and width of the VHC. Macroscopically, we have measured the length of CS, outlined the range of the value and distinguished short and long sinuses. When studying the CS microscopically, we have distinguished two patterns of the CS transverse section.

Our study shows that the length of the CS has had weak correlation with both the length and width of the VHC. In black populations, Ominde et al. (2015) have measured the external length of the heart from the apex to the midpoint between the entry of left and right superior pulmonary veins and got resembling data. The researchers have also revealed that the length of CS showed a positive correlation with that of left atrioventricular groove in both males and females. According to most studies (table 4), the length of the CS varied from 20.5 to $63 \mathrm{~mm}$, the reference range of the average values of the CS length was $33.76-48.4 \mathrm{~mm}$. Only one author has presented larger values (50-80 mm) (Fal'kovskiy, 2014). Taking into account that the author's experience in a large specialized clinical center, it can be assumed that Fal'kovskiy's data characterize the CS in the population of cardiologic patients, especially since there are reports in the literature about a longer CS in heart diseases than without them (Plass et al., 2008; Sun et al., 2012). Kagan et al. (2011) identified three groups of CS: $19.1 \%$ of specimens were long (60-82 mm), 65.4\% were sinuses of medium length (30-59 $\mathrm{mm}$ ) and $15.5 \%$ were short (16-29 mm). Sirajuddin et al. (2020) considered CS with a length of less than $20 \mathrm{~mm}$ as short. The sinuses with a length of over 60 $\mathrm{mm}$, which were not found on our material, can be explained by cardiac pathology (Kagan et al., 2011). The differences in the lengths of the CS may be explained not only by the anatomical variability or heterogeneity of the selected groups, but also by a discrepancy for the beginning of the CS. The beginning of the CS is usually considered to be the confluence of the great cardiac vein and the oblique vein of the LA (Anderson et al., 2013; Chauvin et al., 2000; El-Maasarany et al., 2005;

Table 4. Length of the coronary sinus in human adults: reference values.

\begin{tabular}{|c|c|c|c|c|}
\hline \multirow{2}{*}{ First author (year) } & \multirow{2}{*}{ Methods } & \multirow{2}{*}{ Number of observations } & \multicolumn{2}{|l|}{ Length, mm } \\
\hline & & & $\mathbf{M} \pm \mathbf{S D}$ & Min-max \\
\hline El-Maasarany et al. (2005) & Cad & 40 & $48,4 \pm 5,2$ & ---- \\
\hline Kosourov and Ivanov (2005) & Cad & 200 & ---- & $14-80$ \\
\hline Sousa-Rodrigues et al. (2005) & Cad & 26 & 40,3 & $27-54$ \\
\hline Plass et al. (2008) & $\mathrm{CT}$ & 26 & $108,9 \pm 18$ & $-\cdots--$ \\
\hline Loukas et al. (2009) & Cad \& CT & review & ---- & $20-50$ \\
\hline Ballesteros et al. (2010) & Cad & 68 & $25,96 \pm 6,34$ & ----- \\
\hline Kagan et al. (2011) & CAG (v) & 60 & $46 \pm 15,9$ & $16-82$ \\
\hline Sun et al. (2012) & $\mathrm{CT}$ & 118 & $109 \pm 19$ & $56-159$ \\
\hline Manoranjitham et al. (2015) & Cad & 30 & $54,98 \pm 12,2$ & $-\cdots--$ \\
\hline Ominde et al. (2015) & Cad & 74 & $39,55 \pm 5,32$ & ----- \\
\hline Mehra et al. (2016) & Cad & 40 & $35,35 \pm 4,43$ & $20.9-39.6$ \\
\hline Beegum et al. (2017) & Cad & 50 & $38,22 \pm 8,6$ & $20,5-58,8$ \\
\hline Sharma et al. (2018) & Cad & 30 & $33,76 \pm 5,36$ & $26-48$ \\
\hline Sirajuddin et al. (2019) & $\mathrm{CT}$ & review & ---- & $30-63$ \\
\hline Our present data & Cad & 30 & 34,25 & $13,7-50$ \\
\hline
\end{tabular}

Cad - cadaveric dissection, CT - in vivo computer tomography, CAG (v) - coronary angiography (venous phase). 
Loukas et al., 2009; Sirajuddin et al., 2020), the orifice valve of the great cardiac vein (Vieussen's valve) is usually located there. Beegum et al. (2017) measured CS length from the opening of the oblique vein, and in its absence, from the Vieussen's valve; if the valve was not there, from the confluence of the great cardiac vein with the left marginal vein. Plass et al. (2008) and Sun et al. (2012) understood by CS the entire venous trunk located in the left atrioventricular groove, and this approach determined their data (see Table 4).

In the present study, the minimal and maximal diameters of CS varied over a wide range (Table 2). In order to compare our results with the data of other investigators, we presented mean diameter through the circumference using the formula $\mathrm{L}=$ $2 \pi r$ and the CS diameter varied from 2.4 to 12.8 $\mathrm{mm}$ (Me $5.1 \mathrm{~mm}$ ). In the literature, the diameter of the CS varied from 5-10 mm (Fal'kovskiy, 2014) to $12 \mathrm{~mm}$ (Sirajuddin et al., 2020) or $16 \mathrm{~mm}$ (Loukas et al., 2009). In 63\%, these values were in the range of 9-12 $\mathrm{mm}$ (Sharma et al., 2018). In a study performed on specimens injected with a hardening mass, the diameter of the CS was $8.87 \pm 1.48 \mathrm{~mm}$ (Sharma et al., 2018) and did not differ from the diameter of the CS, measured on macroscopic sections across the CS $(9.3 \pm 5.3 \mathrm{~mm})$ (El-Maasarany et al., 2005), as well as measured intravitally on computed tomograms (Plass et al., 2008). Beegum et al. (2017) found that the width of the CS was $4.25 \pm 1.4 \mathrm{~mm}$ at the origin, increased to $6.98 \pm 2.7 \mathrm{~mm}$ at the opening of the middle cardiac vein and reached maximum values $(9.61 \pm 2.6 \mathrm{~mm})$ in the mouth. Sousa-Rodrigues et al. (2005) have got the same results. The researchers appeared the average diameter of the CS increased from $6.34 \mathrm{~mm}$ to $9.28 \mathrm{~mm}$ from lateral to medial part. Our data of the CS' diameter calculated by means the circumference were lower than the values obtained by El-Maasarany et al. (2005) and Sharma et al. (2018), but in accordance with the results obtained by morphometry of total noninjected specimens (Sousa- Rodrigues et al., 2005, Beegum et al., 2017). With an injection, especially performed without pressure control, the CS could be expanded, and shrinkage of samples is inevitable in the preparation of histological specimens. Nevertheless, taking into account the range of variation of the diameter values, we tend to interpret the revealed differences not as the result of using various techniques, but, first of all, as individual structural features of the CS.

Some sinuses were found to be flattened apicobasally, and in cross-section they seemed to be upright in atrioventricular sulcus, and other CS appeared to lay in the sulcus (Fig. 4a, b). To our knowledge, the latter variant has not been described previously, whereas Jongbloed et al. (2005) have mentioned "upright" sinuses with the oval cross-sectional shape. A flattened sinus should not be considered an artifact for two reasons. First, all specimens were preserved under the same conditions, but some of them showed the CS flattened in superoinferior direction, and in others the CS appeared to be flattened anterior to posterior. Secondly, in microanatomical level, the microenvironment of the two variants of the flattened CS showed no signs of topographic displacement or deformity. Sinuses were uninjected and free of blood, so the obtained data on the cross-section shape of the CS we considered specific for systolic phase.

\section{Myocardium of the coronary sinus}

In this research, we found myocardial sheet within the wall of some sinuses. The striated muscle cuff of the CS was continuous with the left atrial myocardium. Lack of adventitia between the myocardial sheet and tunica media of CS allowed us to consider the sheet as a part of CS wall. Barcelo et al. (2004) delineated the external or free wall of the CS was made up of at least one thin layer of striated myocardial fibers. These fibers were usually seen to start at the level of the entrance of the oblique vein of Marshall. They enveloped the CS in its circumference as a muscular cuff or delicate net (Barcelo et al., 2004). Chauvin et al. (2000) investigated CS in ten human hearts and found a continuous cuff of cardiac striated muscle around the CS. Barcelo et al. (2004) and Chauvin et al. (2000) observed the myocardial coat of CS in every specimen. Our results showed intramyocardial CS as well as CS without myocardial sheet (compare Fig. 6a and Fig. 6b). To our mind, two microanatomical types of CS may be distinguished. The first we propose to term 
myocardialless or, simply, "naked", and the second type we call muscularized. In turn, we propose to divide the latter into partially muscularized (Fig. 5) and completely muscularized (Fig. 6b). When analyzing from the origin to the turning of the CS, in our specimens the myocardium thickened the anterior and posterior wall of the CS, and did not change the thickness of the superior and inferior walls. The thickness of this muscular cuff varied between hearts ( 0.3 to $2.5 \mathrm{~mm}$ ), and in 8 of the 10 hearts it was greater at the level of the ostium $(1.35 \pm 0.7 \mathrm{~mm})$ than at the other end $(0.68 \pm 0.2$ $\mathrm{mm}$ ) (Chauvin et al., 2000). There were no muscle connections between the CS musculature and the left ventricular myocardium (Barcelo et al., 2004). Our data are in accordance with these observations.

\section{Adjacent anatomy of the coronary sinus, left atrium and left fibrous ring}

In our work, lateral half of the CS has been found to be closely to the LFR than its medial part. Mehra et al. (2016) mentioned that the CS was close to the posterior wall of the LA, but the researches did not study adjacent anatomy of those structures. It is known that the course of CS in the atrioventricular sulcus may not be strictly horizontal but may have one or two concavities due to thickening of the muscles of the left ventricular diaphragmatic wall (Fal'kovskiy, 2014). Commonly (49 of 50 cases), the CS laid in atrioventricular sulcus, which is consistent with our results, and only on one specimen the CS was $6.8 \mathrm{~mm}$ higher than the groove (Beegum et al., 2017). Meanwhile, in computed tomography of patients with mitral and aortic valve defects, a high position of the CS was observed in $61.4 \%$ (27 out of 44), in 17 cases out of 44 the CS ran parallel to LFR, located at the same level with the ring. Besides, the CS crossed this ring in six patients (Plass et al., 2008). The distance from the CS to LFR for mitral insufficiency and aortic malformations was $16 \pm 4.1 \mathrm{~mm}$ and $14.2 \pm 3.6 \mathrm{~mm}$ ( $p=0.69)$, respectively (Plass et al., 2008). Sorgente et al. (2008) showed that in vivo and in a large group of patients with normal ventricular function CS lied always behind the posterior wall of the LA at a significant distance from the mitral valvular annulus. In patients with normal dimension of left chambers, the researchers observed a large variability in the distance between CS and mitral annulus. When measuring the distances between CS, LFR and LA, we received a wide variance of the values too. In normal hearts, the distance from the CS to the area of attachment of the mural cusp of the mitral valve to LFR in adults usually varies from 5 to $15 \mathrm{~mm}$ (Anderson et al., 2013), which corresponds with our results. El-Maasarany et al. (2005) evaluated the smallest distance between the CS and LFR at the point of CS origin (9.1 $\mathrm{mm}$ ), and the largest one at its mouth at the right atrium $(11.6 \mathrm{~mm})$. When comparing with these data, in our study, the median of this parameter is slightly smaller $(7.9 \mathrm{~mm})$, and the range of values is wider. The cross-sectional shape of the CS was considered as one of the factors determined these differences. Sinuses which appeared to be flattened in supero-inferior direction lied closely to the LFR than those flattened anteroposteriorly. The intensity of adipose tissue that separates the CS from the posterior wall of the LA can be considered as another factor. After studying the slices made along the CS, it was shown that as the CS approached the right atrium, the width of this fat compartment decreased: it was $1.47 \pm 1.29 \mathrm{~mm}$ in the proximal part, it was $0.95 \pm 0.74 \mathrm{~mm}$ in the middle of the CS, and it was $0.86 \pm 0.55 \mathrm{~mm}$ near the opening (Chauvin et al., 2000). However, when we compared the values of the distances from CS to LFR with these values, it was obvious that the adipose tissue of the coronary groove made an insignificant contribution to the variation of the distances. The third and, in our opinion, the main determine factor for both the gross structural variability and adjacent anatomy of the CS was its myocardial sheet.

\section{Coronary sinus and adjacent arteries}

In this work, we delineated four topographic types for the CS and adjacent arteries. These types have been described and painted earlier in Samoylova's atlas (Samoylova, 1970). When collating these types with the coronary dominance, we showed the first, second and fourth types in the hearts with the right coronary dominance. In the present study, when analyzing histotopograms 
and macroscopic specimens, we found that the arteries were commonly located below the CS. Arteries adjoined CS in 78\% of cadaveric studies (Ortale et al., 2001) and in $90 \%$ of patients (45 of 50 ) who underwent computed tomography (Plass et al., 2008). In $46 \%$ of cases, the CS came into contact with the final part of the RCA, in $32 \%$ it had a contact with the arterial branch from the LCA (Ortale et al., 2001). It is similar to our results. Mehra et al. (2016) found that in 23 of 40 hearts of human adults the circumflex artery was adjacent to the bottom of the terminal part of the great cardiac vein at the CS beginning, but the authors did not specify position of this artery in the remaining 17 hearts (Mehra et al., 2016). Sorgente et al. (2008) found circumflex artery to be located between CS and LFR in 77\% of the patients, but in patients with severe mitral regurgitation CS crossed circumflex artery or marginal branch more frequently, in $97 \%$ of cases. Młynarski et al. (2013) retrospectively analyzed data for 320 patients from 64-slice computed tomography in order to evaluate the CS, circumflex artery and mitral annulus. The researchers found that the CS was usually above the mitral ring (75.9\%), and the circumflex artery was commonly at the level of the posterior part of the ring. The pattern with the artery beneath the CS was also common in our specimens. We have met no hearts where coronary artery ran above the CS. Meanwhile, Kagan and Tyutyunnikova (2017) demonstrated an anatomical specimen with two arterial vessels located above the CS and ensheathing with myocardial loop (Fig. 3b in their article). At the origin of the CS, the circumflex branch of the LCA was located almost at the same frequency above (14 of 45 cases) and below CS (15 of 45 cases); in 16 cases these vessels ran parallel to each other (Plass et al., 2008). The CS crossed with the artery twice or thirds has been regarded as the most dangerous (Młynarski et al., 2013). Our research has not shown such adjacent anatomy.

In conclusion, this study has determined the normative values of the CS length, the values of the CS circumference, as well as the large and small diameters. By means of morphometry, we distinguished short and long CS. We described two variants of the cross-sectional shape of the
CS. Some sinuses appeared to be flattened in the anteroposterior direction whereas the others seemed to be flattened supero-inferiorly. We distinguished two anatomical patterns of the coronary sinus: subepicardial and intramyocardial. The first we proposed to term myocardialless or, simply, "naked", and the second type we named muscularized. We proposed to divide the latter into partially and completely muscularized. Myocardial sleeves we considered to be an integral structure of the intramyocardial CS wall. The results showed the direct dependence of the wall thickness of the CS on the majority of its myocardial cover. We delineated four topographic types for the CS and adjacent arteries, found wide range of variation in the distances from the CS to LA and LFR. The paper presented anatomical patterns of the morphometric adjacent anatomy for the CS that may expand such fundamental biomedical category as "anatomical normality" in cardiac morphology.

\section{REFERENCES}

ANDERSON RH, SPICER D, HLAVACEK AM, COOK AC, BACKER C (2013) Wilcox's surgical anatomy of the heart, $4^{\text {th }}$ ed. Cambridge University Press.

BALLESTEROS LE, RAMÍREZ LM, FORERO PL (2010) Study of the coronary sinus and its tributaries in Colombian subjects. Rev Colomb Cardiol, 17(1): 9-15 (in Spanish).

BEEGUM Z, RAJAN KUMAR S, RAVI KANT SH, NEELAM B (2017) Morphological and morphometric study of coronary sinus in North Indian population. J Clin Diagn Res, 11(9): AC15-AC19.

BARCELO A, DE LA FUENTE LM, STERTZER SH (2004) Anatomic and histologic review of the coronary sinus. Int J Morphol, 22(4): 331-338.

CHAUVIN M, SHAH DC, HAÏSSAGUERRE M, MARCELLIN L, BRECHENMACHER C (2000) The anatomic basis of connections between the coronary sinus musculature and the left atrium in humans. Circulation, 101(6): 647-653.

EL-MAASARANY S, FERRETT CG, FIRTH A, SHEPPARD M, HENEIN MY (2005) The coronary sinus conduit function: Anatomical study (relationship to adjacent structures). Europace, 7(5): 475-481.

FAL'KOVSKIY GE (2014) Structure of the heart and anatomical bases of its function. Bakulev Scientific Centre of Cardiovascular Surgery, Moscow. (in Russian).

HABIB A, LACHMAN N, CHRISTENSEN K.N, ASIRVATHAM SJ (2009) The anatomy of the coronary sinus venous system for the cardiac electrophysiologist. Europace, 11 (suppl 5): v15-v21.

JONGBLOED MR, LAMB HJ, BAX JJ, SCHUIJF JD, DE ROOS A, VAN DER WALL EE, SCHALIJ MJ (2005) Noninvasive visualization of the cardiac venous system using multislice computed tomography. $J$ Am Coll Cardiol, 45: 749-753.

KAGAN II, BELYANIN VV, DEMIN AV (2011) Roentgenoanatomical differences of heart coronary sinus on data of intravital coronary angiography. Morphol Newslett, (3): 39-43 (in Russian).

KAGAN II, TYUTYUNNIKOVA NN (2017) Myocardial bridges and loops as an integral part of topography of heart coronary arteries and their branches. Morfologiia, 152(4): 27-31 (in Russian). 
KOSOUROV AK, IVANOV VA (2005) Structural features of the heart coronary sinus in adult humans. Morfologiia, 128(6): 33-37 (in Russian).

LOUKAS M, BILINSKY S, BILINSKY E, EL-SEDFY A, ANDERSON RH (2009) Cardiac veins: A review of the literature. Clin Anat, 22(1): 129145.

MANORANJITHAM R, SHALINI R, GOSAI SR, RAVI VC (2015) Morphological study of coronary sinus in human cadaveric hearts. Int $J$ Anat Res, 3(3): 1415-1418.

MEHRA L, RAHEJA SH, AGARWAL S, RANI Y, KAUR K, TULI A (2016) Anatomical consideration and potential complications of coronary sinus catheterization. J Clin Diagn Res, 10(2): AC12-AC15.

MŁYNARSKI R, MŁYNARSKA A, SOSNOWSKI M (2013) Anatomical variants of left circumflex artery, coronary sinus and mitral valve can determine safety of percutaneous mitral annuloplasty. Cardiol J, 20(3): 235-240.

OMINDE BS, OLABU B, ONENG'O JA (2015) Length of coronary sinus in a black Kenyan population: correlation with heart length. Anat JAfrica, 4(1): 488-495.

ORTALE JR, GABRIEL EA, IOST C, MÁRQUEZ CQ (2001) The anatomy of the coronary sinus and its tributaries. Surg Radiol Anat, 23: 15-21.

PLASS A, VALENTA I, GAEMPERLI O, KAUFMANN P, ALKADHI H, ZUND G, GRÜNENFELDER J, GENONI M (2008) Assessment of coronary sinus anatomy between normal and insufficient mitral valves by multi-slice computer tomography for mitral annuloplasty device implantation. Eur J Cardiothorac Surg, 33(4): 583-589.

SAMOYLOVA SV (1970) Anatomy of the blood vessels of the heart. Meditsina, Leningrad (in Russian).

SHARMA MK, SINGH J, SHARMA A, KAPOOR K, DEVINDER (2018) Morphology and morphometry of venous drainage system of heart: a retrospective cadaveric study. Int J Cur Res Rev, 10(10): 10-18.

SIRAJUDDIN A, CHEN MY, WHITE CS, ARAI AE (2020) Coronary venous anatomy and anomalies. J Cardiovasc Comput Tomogr, 14(1): 8086.

SORGENTE A, TRUONG QA, CONCA C, SINGH JP, HOFFMANN U, FALETRA FF, KLERSY C, BHATIA R, PEDRAZZINI GB, PASOTTI E, MOCCETTI T, AURICCHIO A (2008) Influence of left atrial and ventricular volumes on the relation between mitral valve annulus and coronary sinus. Am J Cardiol, 102(7): 890-896.

SOUSA-RODRIGUES CF, ALCÂNTARA FS, OLAVE E (2005) Topografía y biometría del sistema venoso coronario y de sus tributaries. Int $J$ Morphol, 23(2): 177-184 (in Spanish).

SUN JP, YANG XS, LAM YY, GARCIA MJ, YU CM (2012) Evaluation of coronary venous anatomy by multislice computed tomography. World $J$ Cardiovasc Surg, 2(4): 91-95. 